\title{
A systematic review investigating the risk of COVID-19 severity and mortality associated with vitamin D sufficiency and deficiency within the adult population
}

\author{
R. Mahmood and S. McLaren \\ School of Human Sciences, London Metropolitan University, London, UK
}

COVID-19 has caused a global pandemic and resulted in 230 million cases and 4.72 million deaths worldwide as of 24 Sep $2021^{(1)}$. Vitamin D (VitD) affects the immune system through the regulation of cathelicidin and Th1 cytokine synthesis. Cathelicidin prevents respiratory illnesses by damaging viral cell membranes ${ }^{(2)}$. COVID-19 infection is associated with an inflammatory cytokine storm. Activated VitD (VDR) receptors down-regulate the synthesis of pro-inflammatory cytokines and increase synthesis of antiinflammatory cytokines ${ }^{(3)}$. VitD may decrease the infection risk of COVID-19 by down-regulating ACE2 receptors ${ }^{(4)}$. Down-regulation of these cytokines by VDRs could mean that VitD can reduce complications associated with COVID-19 ${ }^{(3)}$. Approximately $29 \%$ of the UK adult population is VitD deficient and there is a lack of large-scale randomised control trials (RCT's) on VitD status and COVID-19 mortality and severity ${ }^{(5)}$. Therefore, this systematic review (SR) aimed to assess the risk of COVID-19 mortality and severity according to VitD status within the adult population ( $>18$ years).

Pubmed, Cohrane library, Web of Science and Google Scholar were searched using MeSH terms and keywords relevant to the research question. Studies on vitD status, COVID-19 severity and mortality, published in English, recruiting adults $>18$ years were included. COVID-19 severity was assessed by ICU admission. Study titles and abstracts were screened for inclusion, followed by a full text review according to the preferred reporting items for SR's and meta-analysis (PRISMA) guidelines. A data extraction checklist was used to extract data and conduct statistical analysis on the included studies. Methodological appraisal was undertaken using the checklist of the critical appraisal skills programme (CASP). Publication bias was evaluated using funnel plots. Heterogeneity within the studies was assessed using the $\mathrm{I}^{2}$ statistic. A random-effects model and forest plots were used to evaluate the odds ratio (OR) and thus, estimate the association between VitD deficiency (VDD) or insufficiency and COVID-19 mortality and or severity.

A total of twelve studies with 2458 participants were included in the SR. Overall, the studies showed a low risk of bias through analysis using the CASP tool. The funnel plot for COVID-19 severity was asymmetrical potentially evidencing small-study bias. The studies evidenced moderate heterogeneity $\left(\mathrm{I}^{2}=36 \% \mathrm{p}=0.12\right)$. Therefore, it was appropriate to continue with the meta-analysis. The random- effects analysis found that mortality risk was tripled among VDD or insufficient patients compared with VD sufficient patients $(\mathrm{OR}=2.84,95 \%$ CI 1.83-4.42). Severity of COVID-19 was 3 times higher among VDD or insufficient patients compared with VD sufficient patients (OR $=3.51,95 \%$ CI $2.69-4.57)$.

VDD increases COVID-19 severity and mortality risk among adults. This SR is limited by potential selection bias. Findings are limited to hospitalised patients and cannot be generalised. Further research is warranted to assess the impact of sex on COVID-19 outcomes and larger RCTs are required.

\section{References}

1. WHO Coronavirus (COVID19) Dashboard.

2. Beard JA, Bearden A \& Striker R (2011) J Clin Virol 3, 194-200.

3. Grant WB, Lahore H, McDonnell SL, et al. (2020) Nutrients 4, 988.

4. Hribar CA, Cobbold PH \& Church FC (2020) Brain Sci, 5, 284.

5. Phillips DF (2019) BDA. 